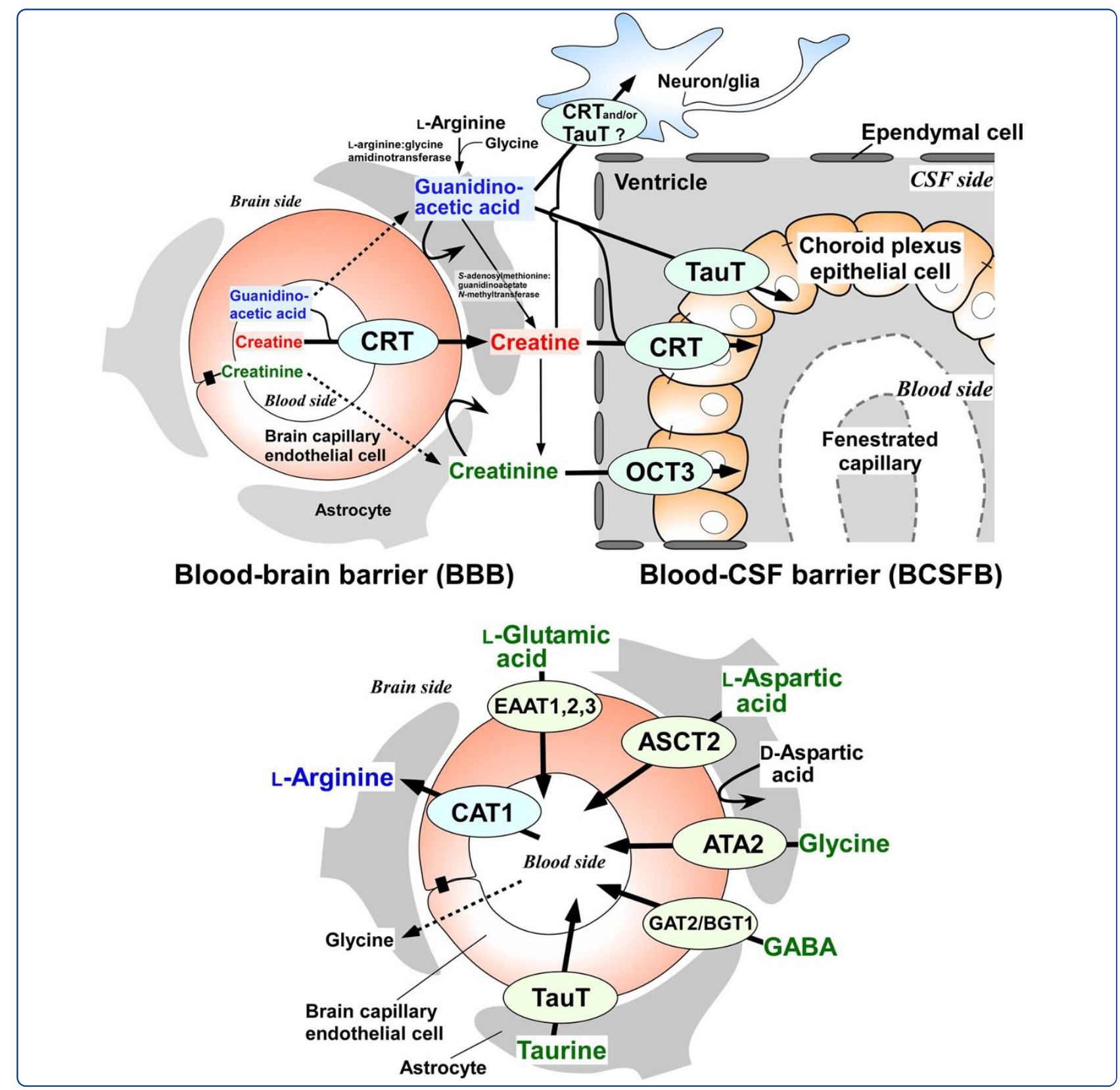

\title{
Transport characteristics of guanidino compounds at the blood-brain barrier and blood-cerebrospinal fluid barrier: relevance to neural disorders
}

Tachikawa and Hosoya 


\title{
Transport characteristics of guanidino compounds at the blood-brain barrier and blood-cerebrospinal fluid barrier: relevance to neural disorders
}

\author{
Masanori Tachikawa, Ken-ichi Hosoya*
}

\begin{abstract}
Guanidino compounds (GCs), such as creatine, phosphocreatine, guanidinoacetic acid, creatinine, methylguanidine, guanidinosuccinic acid, $\gamma$-guanidinobutyric acid, $\beta$-guanidinopropionic acid, guanidinoethane sulfonic acid and $\alpha$ guanidinoglutaric acid, are present in the mammalian brain. Although creatine and phosphocreatine play important roles in energy homeostasis in the brain, accumulation of GCs may induce epileptic discharges and convulsions. This review focuses on how physiologically important and/or neurotoxic GCs are distributed in the brain under physiological and pathological conditions. Transporters for GCs at the blood-brain barrier (BBB) and the blood-cerebrospinal fluid (CSF) barrier (BCSFB) have emerged as substantial contributors to GCs distribution in the brain. Creatine transporter (CRT/solute carrier (SLC) 6A8) expressed at the BBB regulates creatine concentration in the brain, and represents a major pathway for supply of creatine from the circulating blood to the brain. CRT may be a key factor facilitating blood-to-brain guanidinoacetate transport in patients deficient in S-adenosylmethionine: guanidinoacetate $\mathrm{N}$-methyltransferase, the creatine biosynthetic enzyme, resulting in cerebral accumulation of guanidinoacetate. CRT, taurine transporter (TauT/SLC6A6) and organic cation transporter (OCT3/SLC22A3) expressed at the BCSFB are involved in guanidinoacetic acid or creatinine efflux transport from CSF. Interestingly, BBB efflux transport of GCs, including guanidinoacetate and creatinine, is negligible, though the BBB has a variety of efflux transport systems for synthetic precursors of GCs, such as amino acids and neurotransmitters. Instead, the BCSFB functions as a major cerebral clearance system for GCs. In conclusion, transport of GCs at the BBB and BCSFB appears to be the key determinant of the cerebral levels of GCs, and changes in the transport characteristics may cause the abnormal distribution of GCs in the brain seen in patients with certain neurological disorders.
\end{abstract}

\section{Introduction}

Guanidino compounds (GCs), such as creatine (CT), phosphocreatine (PCT), guanidinoacetic acid (GAA), creatinine (CTN), methylguanidine (MG), guanidinosuccinic acid (GSA), $\gamma$-guanidinobutyric acid (GBA), $\beta$-guanidinopropionic acid (GPA), guanidinoethane sulfonic acid (GES) and $\alpha$-guanidinoglutaric acid (GGA), are present in the mammalian brain at individual concentrations in the nanomolar to millimolar range [1-3]. CT and PCT play a pivotal role in the storage and utilization of phosphate-bound energy via CT kinase in the brain, thus serving to maintain energy homeostasis in the brain [4]. In this CT/PCT/CT kinase shuttle system,

\footnotetext{
* Correspondence: hosoyak@pha.u-toyama.ac.jp

Department of Pharmaceutics, Graduate School of Medicine and Pharmaceutical Sciences, University of Toyama, Toyama, Japan
}

the phosphate-bound energy of ATP is transferred to $\mathrm{CT}$ and stored as PCT to be used for regenerating ATP from ADP [4]. On the other hand, GCs are known to be endogenous convulsants [1]. It has been proposed that most GCs are synthesized via transamidination of the amidino group from arginine to amino acids and neurotransmitters [1] (Figure 1). CT biosynthesis involves two sequential steps catalyzed by L-arginine:glycine amidinotransferase (AGAT) and $S$-adenosylmethionine:guanidinoacetate $N$-methyltransferase (GAMT), producing the $\mathrm{CT}$ biosynthetic precursor GAA and CT, respectively. CTN is non-enzymatically produced from CT.

The brain barriers might be key determinants of the levels of GCs in the brain and cerebrospinal fluid (CSF). The brain barriers consist of the blood-brain barrier (BBB) and the blood-cerebrospinal fluid barrier (BCSFB). The $\mathrm{BBB}$ and $\mathrm{BCSFB}$ are formed by tight junctions of

\section{() Biomed Central}




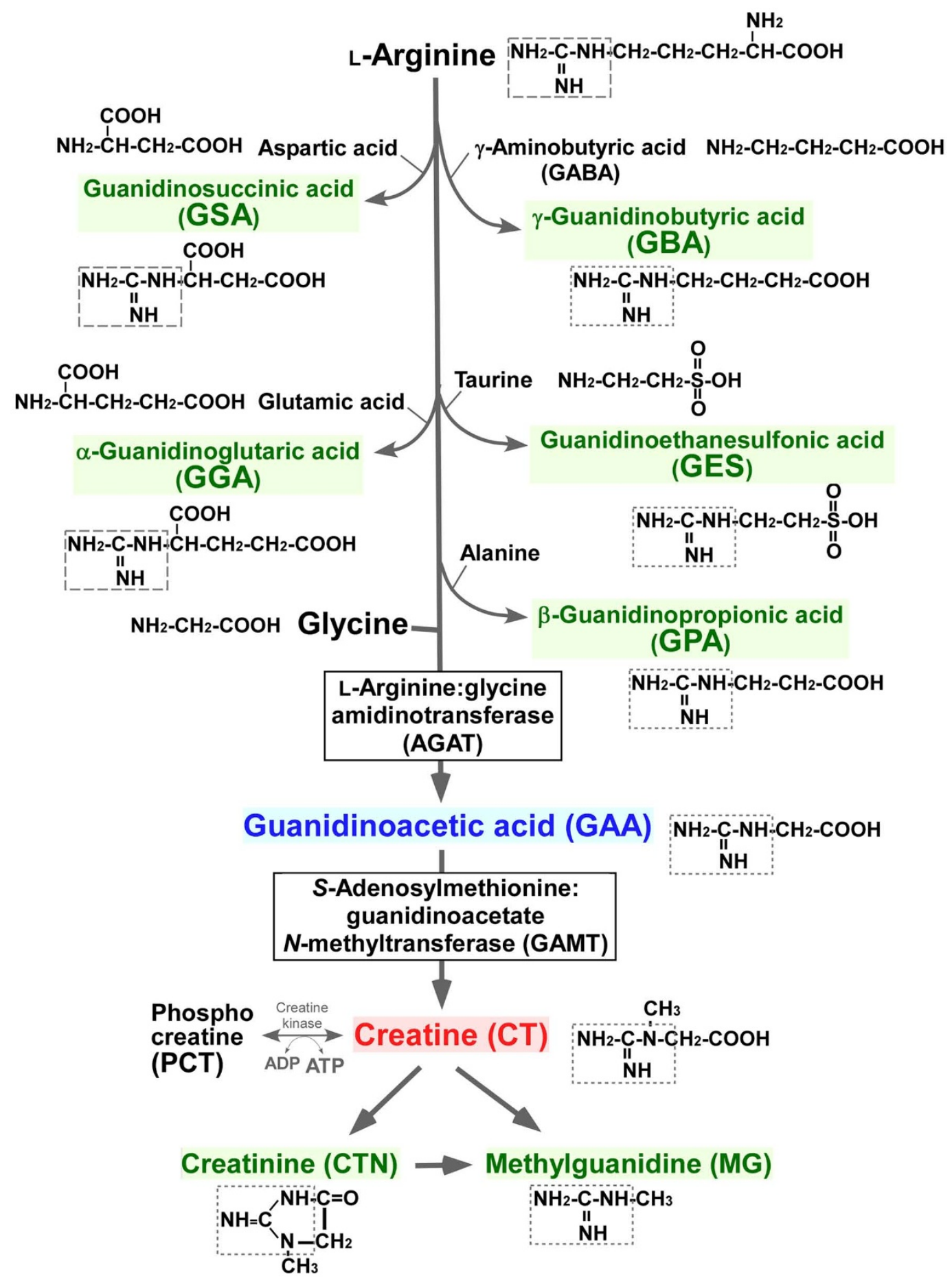

Figure 1 Putative synthetic pathways of guanidino compounds in the brain.

brain capillary endothelial cells and choroid plexus epithelial cells, respectively, and serve to regulate the supply of essential nutrients into the brain/CSF and the elimination of xenobiotics and endogenous metabolites from the brain/CSF via various transport systems such as transporters [5,6]. It is thus conceivable that transporters for GCs play an essential role in the influx and efflux transport of GCs at the BBB and/or the BCSFB. However, evidence on the molecular mechanism(s) of GCs transport between the circulating blood and the brain 
remained incomplete or conflicting. We have investigated the brain barrier transport and cerebral distribution of GCs. In this review, we present an overview of the BBB and the BCSFB transport of GCs and related amino acids and neurotransmitters.

\section{Relevance of GCs transport at the brain barriers to neural disorders}

A physiological importance of CT in the brain has been evidenced in inherited CT deficiency syndrome (CDS), which is characterized by the absence or severe reduction of CT in the brain [7]. Patients exhibit mental retardation, delayed speech and language delay, epilepsy, extrapyramidal signs, and autistic behavior [8]. CDS is associated with genetic defects in AGAT [9,10], GAMT [11], and CT transporter (CRT/solute carrier (SLC) 6A8) [12]. It has been believed that the blood-to-brain CT supply is limited by the BBB, and that cerebral CT is largely derived from biosynthesis in the brain. Indeed, CT has a net positive charge and an estimated log partition coefficient of -2.7 [13], which would not be consistent with diffusion of $\mathrm{CT}$ through plasma membranes. Furthermore, oral administration of $20 \mathrm{~g} \mathrm{CT}$ per day for 4 weeks produced only a $9 \%$ increase in total CT in human brain [14]. On the other hand, in patients with AGAT and GAMT deficiency, oral CT administration increased the CT level in the brain [11] and improved the neurologic symptoms $[9,15]$. However, patients with CRT deficiency do not show improvement of neurological symptoms following oral CT supplementation [16-18], despite the presence of CT biosynthetic enzymes and normal levels of plasma CT. These lines of evidence prompted us to hypothesize that CRT is expressed at the BBB and mediates the transport of $\mathrm{CT}$ from the circulating blood to the brain. To test this idea, it is necessary to identify the origin of $\mathrm{CT}$ in the brain.

Cerebral accumulation of GAA contributes to neurological complications, such as epilepsy and seizures, in patients deficient in GAMT [8]. It has been postulated that GCs cannot undergo active brain-to-blood efflux across the brain barriers, so that cerebral accumulation of GCs may occur. This is consistent with the concept that one of the functions of the BBB is to retain neurotransmitters and nutrients in the brain. Consequently, increased levels of GCs in the brain may be associated with epileptic discharges and convulsions. GAA is commonly found to be elevated in brains having an epileptogenic focus and in CSF of several animal models of epilepsy [1]. In contrast, it has been reported that endogenous levels of GAA in CSF increased at the onset of convulsions but returned to the basal level after the convulsions had ceased [19], supporting the idea that the brain possesses a clearance system for GCs via the brain barriers. It has recently been established that the brain barriers act as a clearance system for various metabolites and neurotoxic compounds produced in the brain [6]. Clarifying the cerebral clearance system of GCs from the brain and CSF should therefore provide insight into how abnormal accumulation of GCs in the brain and CSF can be prevented.

\section{Methodology for evaluation of BBB and BCSFB transport}

The in vivo blood-to-brain and blood-to-CSF influx transports across the BBB and BCSFB have been evaluated by means of integration plot analysis and the brain uptake index (BUI) method after intravenous and carotid artery injection of radiolabeled compounds, respectively [20-22]. This method enables us to calculate the apparent brain/CSF uptake clearance $\left(\mathrm{CL}_{\text {influx, } \mathrm{BBB}}, \mathrm{CL}_{\mathrm{in}-}\right.$ flux, BCSFB). The in vivo brain-to-blood efflux transport clearance $\left(\mathrm{CL}_{\text {efflux, } \mathrm{BBB}}\right)$ across the $\mathrm{BBB}$ has been determined by means of the brain efflux index (BEI) method [23]. After microinjection of a mixture of radiolabeled test and reference compounds into the cerebrum, the remaining amounts of test and reference compounds in the ipsilateral cerebrum are measured. The reference compound, which does not cross the $\mathrm{BBB}$, is used to determine the amount of test compound injected. Apparent efflux clearance reflects the efflux transport process across the $\mathrm{BBB}$, but not across the BCSFB. The in vivo efflux clearance from CSF has been evaluated by means of the intracerebroventricular administration method [24]. After intracerebroventricular administration of a test compound, the remaining amount of test compound in the CSF is determined. The molecular mechanisms responsible for $\mathrm{BBB}$ and BCSFB transport have been investigated by using our established conditionally immortalized rat/mouse brain endothelial cell line (TR/TM-BBB) $[25,26]$, rat choroid plexus epithelial cell line (TR-CSFB) [27] and freshly isolated choroid plexus. TR/TM-BBB cells and TR-CSFB cells retain the in vivo expressions and functions of several transporters and are a suitable in vitro model for the BBB [28] and BCSFB [5], respectively. A number of transporters expressed at the BBB and BCSFB have been identified as playing roles in nutrient supply and drug distribution to the brain and the CSF by using TR/TM-BBB cells and TR-CSFB cells [6].

\section{Transport of GCs precursor amino acids and neurotransmitters across the $\mathrm{BBB}$}

The BBB possesses influx and efflux transport systems for GCs precursor amino acids and neurotransmitters, as illustrated in Figure 2. L-Arginine is transported from the circulating blood into the brain via $\mathrm{Na}^{+}$-independent cationic amino acid transporter 1 (CAT1/SLC7A1) 


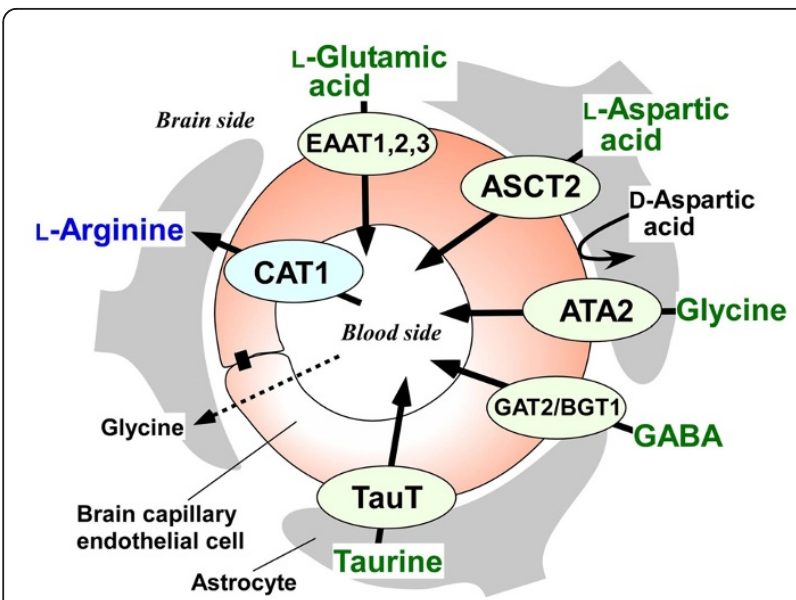

Figure 2 Transport characteristics of guanidino compounds precursor amino acids and neurotransmitters at the BBB.

expressed at the BBB $[29,30]$. The L-arginine influx transport at the rat $\mathrm{BBB}$ is saturable with a MichaelisMenten constant $(\mathrm{Km})$ value of $56 \mu \mathrm{M}$, which is lower than the physiological serum concentration of L-arginine in rat $(170 \mu \mathrm{M}[31])$ and in human $(100 \mu \mathrm{M}$ [32]). Since $\mathrm{L}$-arginine in mammals is derived mostly from renal de novo synthesis and dietary intake, CAT1 at the BBB would function as a supply pathway for L-arginine to the brain. The blood-to-brain transport of glycine across the BBB appears to be a passive diffusion, since $\left[{ }^{14} \mathrm{C}\right] \mathrm{gly}$ cine uptake by the brain was not inhibited by unlabeled glycine [20]. Zlokovic et al. [33] found, using the BUI method, that the BUI value of glycine was close to that of sucrose, used as a BBB impermeable paracellular marker. Since L-serine is possibly converted to glycine in the brain $[34,35]$, glycine in the brain would be supplied by de novo synthesis, rather than via glycine influx transport at the BBB.

L-Glutamic acid, L-aspartic acid, glycine, $\gamma$-aminobutyric acid (GABA), and taurine undergo efflux from the brain into the circulating blood across the BBB [36-39]. A physiological role for the efflux transport processes at the $\mathrm{BBB}$ would be to remove the neurotransmitters and amino acids from the brain interstitial fluid and to maintain these extracellular concentrations below neurotoxic levels [40] if they had overflowed from neurons and glial cells under conditions of cerebral dysfunction, such as brain ischemia.

The brain-to-blood efflux transport across the BBB consists of two steps, i.e., influx across the abluminal membrane from the brain interstitial fluid into brain capillary endothelial cells and subsequent efflux across the luminal membrane from endothelial cells into the circulating blood. $\mathrm{Na}^{+}$-dependent transporters, such as EAAT (excitatory amino acid transporter) 1/SLC1A3,
EAAT2/SLC1A2, and EAAT3/SLC1A1, for glutamate exist on the abluminal membranes of brain capillary endothelial cells [41]. These transporters mediate glutamate transport from the brain interstitial fluid into endothelial cells. When the glutamate concentration in endothelial cells becomes greater than that in the plasma, glutamate would be effluxed from the endothelial cells into the circulating blood in a facilitative manner $[42,43]$. Alanine-serine-cysteine transporter 2 (ASCT2/SLC1A5), mediates L-isomer-selective uptake of aspartic acid on the abluminal membrane of brain capillary endothelial cells in an $\mathrm{Na}^{+}$- and $\mathrm{pH}$-dependent manner [44]. $\mathrm{Na}^{+}$-dependent amino acid transporter A2 (ATA2/SLC38A2) is predominantly expressed at the abluminal membrane of the brain capillary endothelial cells at the BBB and transports system A substrates, such as glycine and L-proline [39]. Mouse $\mathrm{Na}^{+}$-dependent GABA transporter 2/betaine-GABA transporter 1 (GAT2/BGT1/SLC6A12) is expressed in brain capillary endothelial cells and is involved in GABA efflux transport across the BBB [45]. Taurine efflux transport across the BBB is a saturable process with a $\mathrm{Km}$ of $39.1 \mu \mathrm{M}$ [38]. This $\mathrm{Km}$ value is similar to that of rat taurine transporter (TauT/SLC6A6) [46], suggesting that TauT is involved in taurine efflux transport across the BBB.

In contrast to L-glutamic acid, L-aspartic acid, glycine, GABA, and taurine, not only is D-aspartic acid retained in the brain parenchymal cells, but also there is no efflux transport system for D-aspartic acid at the BBB [36]. This would facilitate the accumulation of D-aspartic acid in the brain. While the BBB acts as an efflux pump for L-aspartic acid and L-glutamic acid to reduce the brain interstitial concentration, it acts as a static wall for D-aspartic acid.

\section{Characteristics of transporters for GCs}

Several transporters which belong to the SLC6 gene family are likely to accept GCs as substrates (Table 1). The SLC6 members are $\mathrm{Na}^{+}$- and $\mathrm{Cl}^{-}$-dependent transporters for neurotransmitters, amino acids and osmolytes, including GABA, betaine, taurine, and CT [47]. CRT mediates transport of CT, GAA, and CTN, with $\mathrm{Km}$ values of $46 \mu \mathrm{M} / 29 \mu \mathrm{M}[48,49], 269 \mu \mathrm{M} / 412 \mu \mathrm{M}$ [50], and $52 \mathrm{mM}$ [51], respectively. The transport affinities of GAA and CTN for CRT are one and three orders of magnitude lower than that of CT, respectively. CT competitively inhibits CRT-mediated GAA transport with a Ki value of $60.5 \mu \mathrm{M}$ [50]. Therefore, it is necessary to consider the physiological fluid concentrations of CT and GAA in order to evaluate the actual contribution of CRT to GAA transport. Since CRT is an electrogenic transporter, CT induces an inward current in Xenopus oocytes expressing human CRT at a holding potential of $-60 \mathrm{mV}$ 
Table 1 Inhibitory effect of various compounds on $\left[{ }^{14} \mathrm{C}\right] \mathrm{GAA}$ uptake by HEK293 cells stably overexpressing CRT (CRT/ HEK293 cells) and Xenopus oocytes expressing CRT (CRT/oocytes) or TauT (TauT/oocytes), and $\left[{ }^{14} \mathrm{C}\right] \mathrm{CTN}$ uptake by Xenopus oocytes expressing rOCT3(rOCT3/oocytes)

\begin{tabular}{|c|c|c|c|c|}
\hline \multirow[t]{2}{*}{ Inhibitor } & \multicolumn{3}{|c|}{$\left[{ }^{14} \mathrm{C}\right] \mathrm{GAA}$ uptake } & \multirow{2}{*}{$\begin{array}{c}{\left[{ }^{14} \mathrm{C}\right] \mathrm{CTN} \text { uptake }} \\
\text { rOCT3/oocytes } \\
\% \text { of control }\end{array}$} \\
\hline & $\begin{array}{l}\text { CRT/HEK293 cells } \\
\text { \%of control }\end{array}$ & $\begin{array}{l}\text { CRT/oocytes } \\
\text { \%of control }\end{array}$ & $\begin{array}{l}\text { TauT/oocytes } \\
\text { \%of control }\end{array}$ & \\
\hline Control & $100 \pm 4$ & $100 \pm 9$ & $100 \pm 9$ & $100 \pm 19$ \\
\hline Guanidinoacetate (GAA) & $19.2 \pm 2.6^{*}$ & $44.6 \pm 2.7^{*}$ & $53.2 \pm 9.6^{*}$ & - \\
\hline$\beta$-Guanidinopropionate (GPA) & $1.81 \pm 0.14^{*}$ & $0.944 \pm 0.189^{*}$ & $4.77 \pm 0.66^{*}$ & $143 \pm 21$ \\
\hline$\gamma$-Guanidinobutyrate (GBA) & $10.0 \pm 0.6^{*}$ & $21.4 \pm 2.0^{*}$ & $21.7 \pm 4.2^{*}$ & - \\
\hline Guanidinoethansulfonate (GES) & $23.1 \pm 1.1^{*}$ & - & $4.66 \pm 0.22^{*}$ & - \\
\hline Guanidinosuccinate (GSA) & $101 \pm 1$ & $107 \pm 12$ & $103 \pm 10$ & - \\
\hline Creatine $(C T)$ & $3.06 \pm 0.24^{*}$ & $2.91 \pm 0.23^{*}$ & $98.4 \pm 12.7$ & $137 \pm 24$ \\
\hline Taurine & $111 \pm 5$ & - & $3.00 \pm 0.19^{*}$ & - \\
\hline$\gamma$-Aminobutyric acid (GABA) & $95.5 \pm 2.9$ & - & $34.0 \pm 4.6^{*}$ & - \\
\hline Creatinine (CTN) & $93.3 \pm 5.3$ & $123 \pm 10$ & $97.9 \pm 14.6$ & - \\
\hline Methylguanidine (MG) & $82.3 \pm 15.2$ & $126 \pm 14$ & $110 \pm 18$ & $47.2 \pm 18.9^{* *}$ \\
\hline Creatine phosphate $(\mathrm{PCT})$ & $93.4 \pm 6.8$ & $97.9 \pm 14.1$ & $117 \pm 6$ & - \\
\hline Guanidine & $114 \pm 6$ & $88.2 \pm 13.4$ & - & $137 \pm 22$ \\
\hline L-Arginine & $86.6 \pm 6.3$ & - & - & - \\
\hline Glycine & $126 \pm 4$ & - & - & - \\
\hline L-Alanine & $111 \pm 3$ & - & - & - \\
\hline L-Aspartic acid & $98.5 \pm 2.2$ & - & - & - \\
\hline Tetraethylammonium & $128 \pm 29$ & $82.8 \pm 7.3$ & - & $48.1 \pm 8.3^{* *}$ \\
\hline Benzylpenicillin & $110 \pm 14$ & $115 \pm 12$ & - & - \\
\hline
\end{tabular}

$\left[{ }^{14} \mathrm{C}\right] \mathrm{GAA}(20-45 \mu \mathrm{M})$ and $\left[{ }^{14} \mathrm{C}\right] \mathrm{CTN}(70 \mu \mathrm{M})$ uptake by CRT/oocytes, TauT/oocytes and rOCT3/oocytes was measured at $20^{\circ} \mathrm{C}$ for 60 min in the absence (control) or presence of inhibitors $(2 \mathrm{mM})$. [ $\left.{ }^{14} \mathrm{C}\right] \mathrm{GAA}(3 \mu \mathrm{M})$ uptake by CRT/HEK293 cells was measured at $37^{\circ} \mathrm{C}$ for 5 min in the absence (control) or presence of inhibitors $(2$ $\mathrm{mM}$ ). Each value represents the mean \pm SEM ( $\mathrm{n}=4-8$ for CRT/HEK293 cells, $\mathrm{n}=10-25$ for CRT/oocytes, TauT/oocytes and rOCT3/oocytes). ${ }^{*} \mathrm{p}<0.01,{ }^{* *} \mathrm{p}<0.05$, significantly different from control. -, not determined.

Modified and reproduced from Tachikawa et al. [50,51,56].

[52]. GAA, GPA or GBA at the concentration of $1 \mathrm{mM}$ induces significant inward current in CRT-expressing, but not water-injected, oocytes [50]. In the inhibition study, CRT-mediated GAA transport was significantly inhibited by GES, but not by taurine or GABA [50]. PCT and CTN induce inward current to a lesser extent in CRT-expressing oocytes, while GSA and MG do not induce inward current [50]. Thus, GPA, GBA and GES are potent substrates for CRT, whereas CRT does not recognize GSA, MG, taurine or GABA as a substrate. Dodd and Christie [53] have reported that two or three amino acid substitutions result in the loss of CT transporter activity and gain of a specific GABA transporter function. Because GPA inhibits mouse GABA transporter 3 and 4 (GAT3/SLC6A13 and GAT4/SLC6A11) [54,55], there is a possibility that mouse GAT3 and GAT4 mediate transport of GCs. Therefore, these amino acid residues may be involved in GAT-mediated transport of GCs which are not CRT substrates. TauT accepts taurine, GAA, and GABA as substrates with $\mathrm{Km}$ values of $43 \mu \mathrm{M}$ [46], $215 \mu \mathrm{M}$ [56] and $1.46 \mathrm{mM}$ [57], respectively. The transport affinities of GAA and GABA for TauT are approximately one and two orders of magnitude lower than that of taurine, respectively. TauT-mediated GAA uptake is significantly inhibited by taurine, GABA, GPA, GBA and GES, whereas CT, GSA, MG and CTN have no effect [56]. This result suggests that GPA, GBA and GES are good substrates for TauT, whereas CT, GSA, MG, and CTN are not.

Rat organic cation transporter 3 (rOCT3/SLC22A3) and human OCT2 (hOCT2/SLC22A2) mediate low-affinity CTN transport with $\mathrm{Km}$ values of $47.7 \mathrm{mM}$ [51] and $4.0 \mathrm{mM}$ [58], respectively. In contrast, human OCT1 (hOCT1/SLC22A1) does not recognize CTN [58]. MG inhibits rOCT3-mediated CTN transport [51], implying that MG is also a substrate for rOCT3. Guanidine, GSA and MG inhibit hOCT1 and/or hOCT2mediated tetraethylammonium uptake, while GAA has little effect on the uptake [59]. Thus, the SLC22 members are likely to mediate transport of GSA, CTN, guanidine, and MG, which are not recognized by the SLC6 family members.

\section{Transport of CT across the BBB}

In human brain, the CT concentration $(7.4 \mathrm{mM})$ is approximately 190 -fold greater than that in the plasma 
(39.5 $\mu \mathrm{M})$ [3]. In vivo analysis has revealed that CT is transported from the circulating blood to the brain via a carrier-mediated transport system at the BBB [21]. The $\left[{ }^{14} \mathrm{C}\right] \mathrm{CT}$ influx transport clearance across the BBB was $1.61 \mu \mathrm{L} /(\mathrm{min} \bullet \mathrm{g}$ brain), which is about 6 -fold greater than that of $\left[{ }^{14} \mathrm{C}\right]$ sucrose $[60]$, a BBB impermeable paracellular marker in rats. The apparent cerebrumto-plasma concentration ratio of $\left[{ }^{14} \mathrm{C}\right] \mathrm{CT}$ reaches $30.8 \mathrm{~mL} / \mathrm{g}$ tissue 24 hours after exogenous administration of $\left[{ }^{14} \mathrm{C}\right] \mathrm{CT}$ to mice. This value is consistent with the endogenous brain-to-serum concentration ratio of CT [3], suggesting that the BBB functions as a major pathway for supplying CT to the brain from the circulating blood.

The characteristics of $\left[{ }^{14} \mathrm{C}\right] \mathrm{CT}$ uptake by TM-BBB cells support the idea that CRT is involved in CT transport at the BBB [21]. $\left[{ }^{14} \mathrm{C}\right] \mathrm{CT}$ uptake by TM-BBB cells takes place in an $\mathrm{Na}^{+}-, \mathrm{Cl}^{-}$- and concentration-dependent manner with a $\mathrm{Km}$ value of $16.2 \mu \mathrm{M}$, which is consistent with the apparent $\mathrm{Km}$ values of $29 \mu \mathrm{M}$ for human CRT [61] and $15 \mu \mathrm{M}$ for rat CRT [48]. GPA causes marked inhibition of $\left[{ }^{14} \mathrm{C}\right] \mathrm{CT}$ uptake by TM-BBB cells as reported elsewhere [62]. The corresponding $\mathrm{Km}$ value is 10 - to 40 -fold lower than the plasma concentration (140-600 $\mu \mathrm{M})$ in mouse and rat [2]. The blood-tobrain transport of CT is more than $90 \%$ saturated by endogenous plasma $\mathrm{CT}$, and $\mathrm{CRT}$ at the BBB plays a role in continuously supplying CT from the circulating blood to the brain at a constant rate equivalent to the maximal velocity. This suggests that the efficacy of oral CT treatment for patients with neurodegenerative diseases may depend on the CRT function at the BBB. Belanger et al. [63] have found that the mRNA expression and function of CRT in TM-BBB cells are increased under hyperammonemic conditions. CRT function at the $\mathrm{BBB}$ might be highly regulated under pathophysiological conditions.

Immunohistochemical studies have indicated that CRT is localized at both the luminal and abluminal sides of mouse brain capillary endothelial cells in the adult brain $[21,64]$. CRT is also expressed in neurons [21,65], suggesting that CT is transported into neurons by CRT following $\mathrm{BBB}$ transport. In support of this notion, in vitro studies reveal that an exogenous supply of $\mathrm{CT}$ increases the neuronal PCT store and protects neurons from hypoxic damage, glutamate excitotoxicity and $\beta$-amyloid-induced toxicity $[66,67]$. Oral administration of CT has been reported to protect neurons in animal models of amyotrophic lateral sclerosis, Huntington's disease and Parkinson's disease [68-70]. Thus, the processes of CT transport at the $\mathrm{BBB}$ are important for understanding the mechanism governing the supply of CT to the brain and information about them could help in the design of improved oral CT supplementation for the treatment of neurodegenerative diseases and CDS.

\section{Transport of GAA across the BBB and BCSFB}

GAA is present at low concentrations $(2-12 \mu \mathrm{M})$ in normal mammalian brain [3]. In contrast, the GAA level in the brain of GAMT-knockout mice (1.85-1.87 $\mathrm{mM}$ ) is approximately 150 -fold greater than that in normal brain $(12 \mu \mathrm{M})$ [71,72]. Since GAA exerts an epileptogenic effect by affecting GABAergic neurotransmission [73], an increased level of GAA in the brain appears to affect brain function. We have investigated why GAA is abnormally accumulated in the brains of patients with GAMT deficiency.

\section{Brain-to-blood efflux transport of GAA across the BBB and BCSB}

In vivo and ex vivo studies reveal that there is no efflux transport of GAA across the BBB, and instead GAA undergoes efflux transport from the CSF $[50,56]$. $\left[{ }^{14} \mathrm{C}\right]$ GAA microinjected into rat cerebrum was not effluxed from the brain across the $\mathrm{BBB}$ [56], whereas the elimination clearance of GAA via the BCSFB $(3.97 \mu \mathrm{L} / \mathrm{min}$ per rat) [50] is still approximately 33 -fold greater than the apparent blood-to-CSF influx clearance of GAA $(0.122 \mu \mathrm{L} / \mathrm{min}$ per rat) [56]. This predominant GAA efflux transport at the BCSFB can explain the fact that the GAA concentration in human CSF $(0.036-0.22 \mu \mathrm{M})$ is almost one order of magnitude lower than that in plasma $(0.35-3.5 \mu \mathrm{M})$ [8]. This also may help to explain the rapid removal of increased GAA from the CSF at the onset of convulsions [19], preventing the continuation of convulsions and seizures.

GAA transport at the BCSFB is mediated at least partially by CRT and TauT, which may make approximately $50 \%$ and $11 \%$ contributions to total GAA uptake by the choroid plexus, respectively $[50,56]$. The functional expression of CRT at the BCSFB is supported by the brush-border membrane localization of CRT in choroid plexus epithelial cells and the observation of $\mathrm{Na}^{+}$- and $\mathrm{Cl}^{-}$-dependent $\left[{ }^{14} \mathrm{C}\right] \mathrm{CT}$ uptake by TR-CSFB [50]. TauT appears to be expressed on the brush-border membrane of choroid plexus epithelial cells [74]. The $\mathrm{Km}$ value of $\mathrm{Na}^{+}$-dependent GAA uptake by TR-CSFB cells (929 $\mu \mathrm{M})$ is comparable with that of GAA uptake by CRTexpressing HEK293 cells $(412 \mu \mathrm{M}) /$ Xenopus laevis oocytes $(269 \mu \mathrm{M})$, as well as that of TauT-expressing Xenopus laevis oocytes $(215 \mu \mathrm{M})[50,56]$. The in vivo process of GAA elimination from the CSF was partially inhibited by CT and taurine $[50,56]$.

Interestingly, one clinical report has demonstrated that patients with CRT deficiency exhibit increased GAA levels, as well as the absence of CT in the brain [75], 
implying a causal relationship between the gene defect of CRT and the increase in brain GAA level. Although neurological complications in patients with CRT deficiency would be caused mainly by the lack of CT transport in neuronal cells, the dysfunction of CRT may also lead to an increase in GAA in the brain, owing to the lack of CRT-mediated GAA efflux transport across the BCSFB. In patients with GAMT deficiency, GAA is accumulated in the CSF (11-15 $\mu \mathrm{M})$, whereas CT levels in the CSF are significantly decreased $(<2.0 \mu \mathrm{M})$. However, GAA concentration in the CSF of these patients is still lower than that in the brain parenchyma [76] or plasma [8]. This is because CRT and TauT would play an important role in the efflux transport of GAA from the CSF at the BCSFB. Indeed, the $\mathrm{Km}$ value of GAA uptake by TR-CSFB cells $(926 \mu \mathrm{M})[50]$ is greater than the GAA concentration in the CSF of the patients (11-15 $\mu \mathrm{M}$ [76]).

\section{Blood-to-brain influx transport of GAA across the BBB}

GAA influx transport via CRT at the BBB would also play a key role in the cerebral accumulation of GAA in patients with GAMT deficiency. The characteristics of $\mathrm{Na}^{+}$- and $\mathrm{Cl}^{-}$-dependent $\left[{ }^{14} \mathrm{C}\right] \mathrm{GAA}$ uptake by TR-BBB cells are consistent with those of CRT, but not TauT [50]. The $\mathrm{Km}$ value of $556 \mu \mathrm{M}$ in TR-BBB cells is comparable with that obtained for GAA uptake using CRTexpressing Xenopus laevis oocytes $(269 \mu \mathrm{M}) / \mathrm{HEK} 293$ cells $(412 \mu \mathrm{M})$. Taurine has no significant effect on the GAA uptake. CRT is localized in mouse brain capillary endothelial cells and mediates CT influx transport across the BBB $[21,64]$. Considering that the $\mathrm{Km}$ value of rat CRT-mediated CT transport is $46 \mu \mathrm{M} / 29 \mu \mathrm{M}$ [48,49], the function of CRT on the luminal membrane could be 85 90\% saturated by endogenous CT in the serum under physiological conditions $(250 \mu \mathrm{M}$ in rats [3]). Thus, it appears that CRT-mediated transport of GAA at the BBB is almost wholly inhibited by CT in the blood under normal conditions. In patients with GAMT deficiency, the CT levels in the plasma $(1-5 \mu \mathrm{M})$ are smaller than reference values (6-109 $\mu \mathrm{M})$, whereas the GAA levels in the plasma (12-39 $\mu \mathrm{M})$ are greater than reference values (0.35-3.5 $\mu \mathrm{M})$ [8]. Although the influx and efflux transport of GAA across the brain barriers are well balanced, at least under normal conditions [56], the degree of CRT saturation by endogenous $\mathrm{CT}$ in the circulating blood of the patients would be smaller, thus contributing to the significant increase of the blood-to-brain GAA transport. This mechanism could explain the fact that treatment with CT supplementation resulted in partial normalization of cerebral levels of CT and GAA in patients [77].

\section{GAA transport in brain parenchymal cells}

$\left[{ }^{14} \mathrm{C}\right] \mathrm{GAA}$ is taken up by brain parenchymal cells in a concentrative manner, presumably via CRT, TauT and other $\mathrm{Na}^{+}$-dependent carrier-mediated system(s) [56]. This would be another reason for the accumulation of GAA in the brain of patient with GAMT deficiency. The contribution of CRT to the GAA uptake by brain parenchymal cells appears to be small, because CT does not inhibit the GAA uptake by more than $11 \%$ even at a concentration of $10 \mathrm{mM}$. It has been reported that there is a relationship between gene polymorphism of CRT, transport activity of CRT, and neurological symptoms such as developmental delay, mental disorder and epilepsy with seizures [78-80]. Almeida et al. [81] proposed that exocytotic release of CT into the brain interstitial fluid acts as a neuromodulator of GABAergic neurotransmission. Thus, CRT may be mainly responsible for the uptake of CT by CRT-expressing neurons and oligodendrocytes to maintain the proper GABAergic neurotransmission. GPA inhibits the GAA uptake by brain slices by $89 \%$, which is greater than the inhibitory effect of taurine plus CT (59\%), but similar to the effect of $\mathrm{Na}^{+}$-depletion (92\%) [56]. Since GPA is a potent inhibitor of mouse GAT3 and GAT4 [54,55], as well as of CRT- and TauT-mediated GAA uptake (Table 1), there is a possibility that the GATs play a role in GAA uptake.

In summary, the key factors facilitating GAA accumulation in the brains of patients with GAMT deficiency could be (i) the lack of efflux transport across the BBB, (ii) the increase of CRT-mediated blood-to-brain GAA transport at the $\mathrm{BBB}$, and (iii) the $\mathrm{Na}^{+}$-dependent concentrative GAA transport by brain parenchymal cells.

\section{Transport of CTN across the BBB and BCSFB}

Several GCs, such as CTN, MG, guanidine, and GSA, are involved in the typical manifestations, such as epileptic and cognitive symptoms, of uremic encephalopathy [82]. Levels of uremic GCs are substantially increased in serum, CSF, and brain of nondialyzed patients with renal insufficiency $[83,84]$. The highest CTN concentration in the CSF of severely uremic patients is $521 \mu \mathrm{M}$, which is about 8-fold greater than that in control subjects $(67.6 \mu \mathrm{M})$ [82]. The uremic GC concentrations observed in the brain of uremic patients are similar to those producing convulsive effects [82], and so could contribute to the neurological complications suffered by these patients [85]. Since no specific saturable uptake mechanism exists for CTN in human red blood cells [86], it has been postulated that CTN diffuses out of the brain. In the kidney, CTN is excreted into urine by tubular secretion, as well as glomerular filtration. Human OCT2, but not hOCT1, mediates the tubular secretion of CTN at the basolateral membrane of renal proximal tubular cells [58]. These findings pose a question as to whether CTN undergoes efflux from the brain only by diffusion. 


\section{Brain-to-blood efflux transport of CTN across the BBB and BCSFB}

Emerging evidence indicates that the BCSFB is the major pathway of cerebral CTN clearance, and transporter-mediated processes, at least in part via rOCT3, are involved in CTN efflux transport from the CSF at the BCSFB [51]. CTN, after intracerebral administration, is not significantly eliminated from the brain across the $\mathrm{BBB}$, whereas the elimination clearance of CTN from the CSF is 60-fold greater than that of inulin (reflecting CSF bulk flow). Even in renal failure model rats, the ratio of increase of the CTN concentration in CSF was smaller than that in plasma, suggesting a significant role for the CSF-to-blood efflux process. The inhibitory effects of inhibitors and antisense oligonucleotides on $\left[{ }^{14} \mathrm{C}\right] \mathrm{CTN}$ uptake by isolated choroid plexus indicate the involvement of rOCT3 and CRT in CTN transport. Rat OCT3 is functionally expressed in choroid plexus epithelial cells [87]. Since endogenous compounds in the CSF may affect rOCT3- and CRT-mediated transport of CTN in the choroid plexus, the physiological conditions in the CSF should be taken into account in evaluating the actual contributions of rOCT3 and CRT. Considering the $\mathrm{Km}$ value of CRT for CT $(46 \mu \mathrm{M} / 29$ $\mu \mathrm{M}[48,49])$ and the normal CSF concentration of CT (24-66 $\mu \mathrm{M}$ [8]), CRT on the brush-border membrane of the choroid plexus epithelial cells may be approximately $50 \%$ saturated by endogenous CT in the CSF. Furthermore, the $\mathrm{Km}$ value of CRT for CTN (52 $\mathrm{mM}$ ) [51] is almost 3 orders of magnitude greater than the $\mathrm{Km}$ value of CRT for CT. Thus, it is unlikely that CRT plays a role in the elimination of CTN from the CSF. Therefore, it appears that CTN efflux transport, at least in part via rOCT3, at the BCSFB plays a crucial role in the efficient removal of CTN from the CSF. Indeed, the $\mathrm{Km}$ value of rOCT3-mediated CTN transport (47.7 $\mathrm{mM}$ ) [51] is still much higher than the CTN concentration in the CSF of patients with renal failure (168-521 $\mu \mathrm{M}$ [85]), suggesting that $\mathrm{rOCT} 3$ functions as a CTN transporter without saturation.

\section{Blood-to-brain influx transport of CTN across the BBB}

The blood-to-brain influx clearance of CTN is approximately 14-fold greater than that of sucrose, used as a BBB impermeable paracellular marker [60]. This evidence implies that the blood-to-brain transport of CTN is carrier-mediated, rather than occurring simply by passive diffusion. In this regard, elevated levels of CTN in the brain of the patients with renal insufficiency might be due to increased permeability of CTN in the bloodto-brain direction. Therefore, it will be clinically beneficial, as far as the cerebral accumulation of CTN is concerned, to block the BBB influx transport of CTN in patients with renal insufficiency.

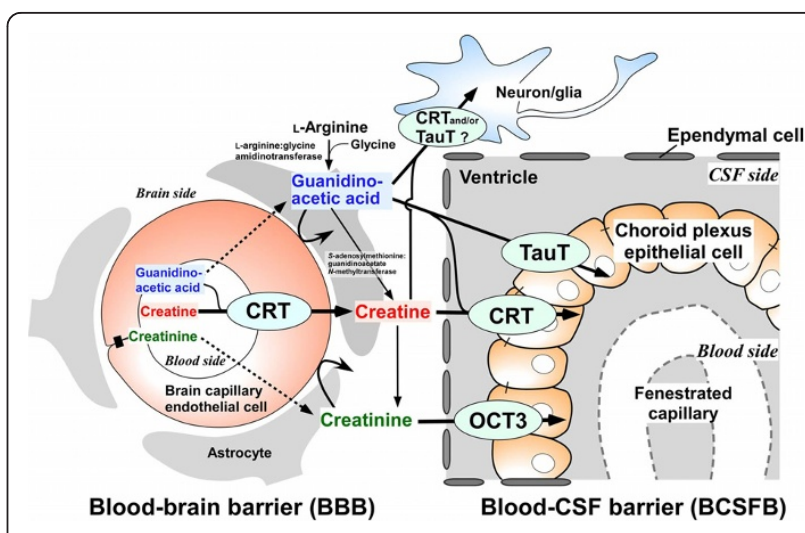

Figure 3 Transport characteristics of guanidino compounds at the BBB and the BSCFB.

\section{Conclusion}

Our recent research provides a novel insight into how physiologically important and/or neurotoxic GCs are accumulated in the brain under physiological and pathological conditions. Transporters for GCs at the BBB and BCSFB have emerged as determinants of GCs distribution to the brain as summarized in Figure 3. CRT expressed at the $\mathrm{BBB}$ regulates the $\mathrm{CT}$ concentration in the brain at millimolar levels as a major pathway for supplying CT from the circulating blood to the brain. CRT may also be a key factor in blood-to-brain GAA transport in patients with GAMT deficiency, causing abnormal accumulation of GAA in the brain. CRT, TauT and OCT3 expressed at the BCSFB are involved in GAA and/or CTN efflux transport from the CSF. Interestingly, $\mathrm{BBB}$ efflux transport of $\mathrm{GCs}$, including GAA and CTN, is negligible, though the BBB has a variety of efflux transport systems for synthetic precursors of GCs, such as amino acids and neurotransmitters. Instead, the BCSFB functions as a major cerebral clearance system for GCs. These findings should help us identify the molecular mechanism(s) of neurological complications in unexplained convulsions and seizures. The regulatory mechanisms of GCs transport at the $\mathrm{BBB}$ and $\mathrm{BCSFB}$ need to be further clarified to provide a more rational basis for therapy of neuronal disorders caused by accumulation of GCs and insufficiency of CT in the brain. Such information should also aid the discovery and development of drugs which facilitate efflux transport of GCs at the BBB and the BCSFB in order to prevent accumulation of GCs, as well as drugs which facilitate the influx transport of $\mathrm{CT}$ at the $\mathrm{BBB}$ to promote efficient supplementation of CT.

\section{Abbreviations}

AGAT: L-arginine:glycine amidinotransferase; ASCT: alanine-serine-cysteine transporter; ATA: amino acid transporter A; BBB: blood-brain barrier; BCSFB: blood-cerebrospinal fluid barrier; BEl: brain efflux index; BGT: betaine- $\gamma$ - 
aminobutyric acid transporter; BUI: brain uptake index; CAT: cationic amino acid transporter; CDS: creatine deficiency syndrome; CRT: creatine transporter; CT: creatine; CTN: creatinine; CSF: cerebrospinal fluid; EAAT: excitatory amino acid transporter; GAA: guanidinoacetic acid; GABA: $\gamma^{-}$ aminobutyric acid; GAMT: S-adenosylmethionine:guanidinoacetate $\mathrm{N}$ methyltransferase; GAT: $\gamma$-aminobutyric acid transporter; GBA: $\gamma$ guanidinobutyric acid; GC: guanidino compound; GES: guanidinoethane sulfonic acid; GGA: a-guanidinoglutaric acid; GPA: $\beta$-guanidinopropionic acid; GSA: guanidinosuccinic acid; MG: methylguanidine; OCT: organic cation transporter; PCT: phosphocreatine; SLC: solute carrier; TauT: taurine transporter;

\section{Acknowledgements}

The authors gratefully acknowledge Drs. T. Terasaki and M. Watanabe, and S. Akanuma for their insights and contributions to ongoing research in the authors' laboratory. This study was supported, in part, by a Grant-in Aid for Scientific Research from the Ministry of Education, Science, Sports, and Culture, and a grant from the Japan Society for the Promotion of Science, Japan.

\section{Authors' contributions}

All authors contributed to the writing of this review. All authors have read and approved the final version of the manuscript.

\section{Competing interests}

The authors declare that they have no competing interests.

Received: 30 December 2010 Accepted: 28 February 2011

Published: 28 February 2011

\section{References}

1. Hiramatsu M: A role for guanidino compounds in the brain. $\mathrm{Mol}$ Cell Biochem 2003, 244:57-62.

2. Marescau B, De Deyn P, Wiechert P, Van Gorp L, Lowenthal A: Comparative study of guanidino compounds in serum and brain of mouse, rat, rabbit, and man. J Neurochem 1986, 46:717-720.

3. Marescau B, Deshmukh DR, Kockx M, Possemiers I, Qureshi IA, Wiechert P, De Deyn PP: Guanidino compounds in serum, urine, liver, kidney, and brain of man and some ureotelic animals. Metabolism 1992, 41:526-532.

4. Wyss M, Kaddurah-Daouk R: Creatine and creatinine metabolism. Physiol Rev 2000, 80:1107-1213.

5. Hosoya K, Hori S, Ohtsuki S, Terasaki T: A new in vitro model for bloodcerebrospinal fluid barrier transport studies: an immortalized choroid plexus epithelial cell line derived from the tsA58 SV40 large T-antigen gene transgenic rat. Adv Drug Deliv Rev 2004, 56:1875-1885.

6. Ohtsuki S, Terasaki T: Contribution of carrier-mediated transport systems to the blood-brain barrier as a supporting and protecting interface for the brain; importance for CNS drug discovery and development. Pharm Res 2007, 24:1745-1758.

7. Stockler S, Schutz PW, Salomons GS: Cerebral creatine deficiency syndromes: clinical aspects, treatment and pathophysiology. Subcell Biochem 2007, 46:149-166.

8. Almeida LS, Verhoeven NM, Roos B, Valongo C, Cardoso ML, Vilarinho L, Salomons GS, Jakobs C: Creatine and guanidinoacetate: diagnostic markers for inborn errors in creatine biosynthesis and transport. Mol Genet Metab 2004, 82:214-219.

9. Bianchi MC, Tosetti M, Fornai F, Alessandri MG, Cipriani P, De Vito G, Canapicchi R: Reversible brain creatine deficiency in two sisters with normal blood creatine level. Ann Neurol 2000, 47:511-513.

10. Item CB, Stockler-Ipsiroglu S, Stromberger C, Muhl A, Alessandri MG, Bianchi MC, Tosetti M, Fornai F, Cioni G: Arginine:glycine amidinotransferase deficiency: the third inborn error of creatine metabolism in humans. Am J Hum Genet 2001, 69:1127-1133.

11. Stockler S, Holzbach U, Hanefeld F, Marquardt I, Helms G, Requart M, Hanicke W, Frahm J: Creatine deficiency in the brain: a new, treatable inborn error of metabolism. Pediatr Res 1994, 36:409-413.

12. Salomons GS, van Dooren SJ, Verhoeven NM, Cecil KM, Ball WS, Degrauw TJ, Jakobs C: X-linked creatine-transporter gene (SLC6A8) defect: a new creatine-deficiency syndrome. Am J Hum Genet 2001, 68:1497-1500
13. Persky AM, Brazeau GA: Clinical pharmacology of the dietary supplement creatine monohydrate. Pharmacol Rev 2001, 53:161-176.

14. Dechent P, Pouwels PJ, Wilken B, Hanefeld F, Frahm J: Increase of total creatine in human brain after oral supplementation of creatinemonohydrate. Am J Physiol 1999, 277:R698-704.

15. Stockler S, Hanefeld F, Frahm J: Creatine replacement therapy in guanidinoacetate methyltransferase deficiency, a novel inborn error of metabolism. Lancet 1996, 348:789-790.

16. Bizzi A, Bugiani M, Salomons GS, Hunneman DH, Moroni I, Estienne M, Danesi U, Jakobs C, Uziel G: X-linked creatine deficiency syndrome: a novel mutation in creatine transporter gene SLC6A8. Ann Neurol 2002, 52:227-231.

17. Cecil KM, Salomons GS, Ball WS Jr, Wong B, Chuck G, Verhoeven NM, Jakobs C, DeGrauw TJ: Irreversible brain creatine deficiency with elevated serum and urine creatine: a creatine transporter defect? Ann Neurol 2001, 49:401-404.

18. Poo-Arguelles P, Arias A, Vilaseca MA, Ribes A, Artuch R, Sans-Fito A, Moreno A, Jakobs C, Salomons G: X-Linked creatine transporter deficiency in two patients with severe mental retardation and autism. $J$ Inherit Metab Dis 2006, 29:220-223.

19. Hiramatsu M, Edamatsu R, Fujikawa N, Shirasu A, Yamamoto M, Suzuki S, Mori A: Measurement during convulsions of guanidino compound levels in cerebrospinal fluid collected with a catheter inserted into the cisterna magna of rabbits. Brain Res 1988, 455:38-42.

20. Hosoya K, Yamamoto A, Akanuma S, Tachikawa M: Lipophilicity and transporter influence on blood-retinal barrier permeability: a comparison with blood-brain barrier permeability. Pharm Res 2010, 27:2715-2724.

21. Ohtsuki S, Tachikawa M, Takanaga H, Shimizu H, Watanabe M, Hosoya K, Terasaki T: The blood-brain barrier creatine transporter is a major pathway for supplying creatine to the brain. $J$ Cereb Blood Flow Metab 2002, 22:1327-1335.

22. Tachikawa M, Hosoya K, Ohtsuki S, Terasaki T: A novel relationship between creatine transport at the blood-brain and blood-retinal barriers, creatine biosynthesis, and its use for brain and retinal energy homeostasis. Subcell Biochem 2007, 46:83-98.

23. Kakee A, Terasaki T, Sugiyama Y: Brain efflux index as a novel method of analyzing efflux transport at the blood-brain barrier. J Pharmacol Exp Ther 1996, 277:1550-1559.

24. Kitazawa T, Hosoya K, Takahashi T, Sugiyama Y, Terasaki T: In-vivo and invitro evidence of a carrier-mediated efflux transport system for oestrone-3-sulphate across the blood-cerebrospinal fluid barrier. J Pharm Pharmacol 2000, 52:281-288.

25. Hosoya K, Tetsuka K, Nagase K, Tomi M, Saeki S, Ohtsuki S, Takanaga H, Yanai N, Obinata M, Kikuchi A, et al: Conditionally immortalized brain capillary endothelial cell lines established from a transgenic mouse harboring temperature-sensitive simian virus 40 large T-antigen gene. AAPS PharmSci 2000, 2:E27.

26. Hosoya K, Takashima T, Tetsuka K, Nagura T, Ohtsuki S, Takanaga H, Ueda M, Yanai N, Obinata M, Terasaki T: mRna expression and transport characterization of conditionally immortalized rat brain capillary endothelial cell lines; a new in vitro BBB model for drug targeting. J Drug Target 2000, 8:357-370.

27. Kitazawa T, Hosoya K, Watanabe M, Takashima T, Ohtsuki S, Takanaga H, Ueda M, Yanai N, Obinata M, Terasaki T: Characterization of the amino acid transport of new immortalized choroid plexus epithelial cell lines: a novel in vitro system for investigating transport functions at the bloodcerebrospinal fluid barrier. Pharm Res 2001, 18:16-22.

28. Terasaki T, Ohtsuki S, Hori S, Takanaga H, Nakashima E, Hosoya K: New approaches to in vitro models of blood-brain barrier drug transport. Drug Discov Today 2003, 8:944-954.

29. O'Kane RL, Vina JR, Simpson I, Zaragoza R, Mokashi A, Hawkins RA: Cationic amino acid transport across the blood-brain barrier is mediated exclusively by system y+. Am J Physiol Endocrinol Metab 2006, 291 E412-419.

30. Stoll J, Wadhwani KC, Smith QR: Identification of the cationic amino acid transporter (System y+) of the rat blood-brain barrier. J Neurochem 1993, 60:1956-1959.

31. Aoyagi K: Inhibition of arginine synthesis by urea: a mechanism for arginine deficiency in renal failure which leads to increased hydroxyl radical generation. Mol Cell Biochem 2003, 244:11-15. 
32. Kamada $Y$, Nagaretani H, Tamura S, Ohama T, Maruyama T, Hiraoka H, Yamashita S, Yamada A, Kiso S, Inui Y, et al: Vascular endothelial dysfunction resulting from L-arginine deficiency in a patient with lysinuric protein intolerance. J Clin Invest 2001, 108:717-724.

33. Zlokovic BV, Begley DJ, Chain DG: Blood-brain barrier permeability to dipeptides and their constituent amino acids. Brain Res 1983, 271:65-71

34. Furuya S, Watanabe M: Novel neuroglial and glioglial relationships mediated by L-serine metabolism. Arch Histol Cytol 2003, 66:109-121.

35. Yang JH, Wada A, Yoshida K, Miyoshi Y, Sayano T, Esaki K, Kinoshita MO, Tomonaga S, Azuma N, Watanabe M, et al: Brain-specific Phgdh Deletion Reveals a Pivotal Role for L-Serine Biosynthesis in Controlling the Level of D-Serine, an N-methyl-D-aspartate Receptor Co-agonist, in Adult Brain. J Biol Chem 2010, 285:41380-41390.

36. Hosoya K, Sugawara M, Asaba H, Terasaki T: Blood-brain barrier produces significant efflux of L-aspartic acid but not D-aspartic acid: in vivo evidence using the brain efflux index method. J Neurochem 1999, 73:1206-1211.

37. Kakee A, Takanaga H, Terasaki T, Naito M, Tsuruo T, Sugiyama Y: Efflux of a suppressive neurotransmitter, GABA, across the blood-brain barrier. J Neurochem 2001, 79:110-118.

38. Lee NY, Kang YS: The brain-to-blood efflux transport of taurine and changes in the blood-brain barrier transport system by tumor necrosis factor-alpha. Brain Res 2004, 1023:141-147.

39. Takanaga H, Tokuda N, Ohtsuki S, Hosoya K, Terasaki T: ATA2 is predominantly expressed as system $A$ at the blood-brain barrier and acts as brain-to-blood efflux transport for L-proline. Mol Pharmacol 2002, 61:1289-1296.

40. Kanai Y: Family of neutral and acidic amino acid transporters: molecular biology, physiology and medical implications. Curr Opin Cell Biol 1997, 9:565-572.

41. O'Kane RL, Martinez-Lopez I, DeJoseph MR, Vina JR, Hawkins RA: Na (+)-dependent glutamate transporters (EAAT1, EAAT2, and EAAT3) of the blood-brain barrier. A mechanism for glutamate removal. J Biol Chem 1999, 274:31891-31895

42. Benrabh H, Lefauconnier JM: Glutamate is transported across the rat blood-brain barrier by a sodium-independent system. Neurosci Lett 1996, 210:9-12.

43. Lee WJ, Hawkins RA, Vina JR, Peterson DR: Glutamine transport by the blood-brain barrier: a possible mechanism for nitrogen removal. Am J Physiol 1998, 274:C1101-1107

44. Tetsuka K, Takanaga H, Ohtsuki S, Hosoya K, Terasaki T: The I-isomerselective transport of aspartic acid is mediated by ASCT2 at the bloodbrain barrier. J Neurochem 2003, 87:891-901.

45. Takanaga H, Ohtsuki S, Hosoya K, Terasaki T: GAT2/BGT-1 as a system responsible for the transport of gamma-aminobutyric acid at the mouse blood-brain barrier. J Cereb Blood Flow Metab 2001, 21:1232-1239.

46. Smith KE, Borden LA, Wang CH, Hartig PR, Branchek TA, Weinshank RL: Cloning and expression of a high affinity taurine transporter from rat brain. Mol Pharmacol 1992, 42:563-569.

47. Chen NH, Reith ME, Quick MW: Synaptic uptake and beyond: the sodiumand chloride-dependent neurotransmitter transporter family SLC6. Pflugers Arch 2004, 447:519-531.

48. Saltarelli MD, Bauman AL, Moore KR, Bradley CC, Blakely RD: Expression of the rat brain creatine transporter in situ and in transfected HeLa cells. Dev Neurosci 1996, 18:524-534.

49. Schloss $P$, Mayser $W$, Betz $H$ : The putative rat choline transporter CHOT1 transports creatine and is highly expressed in neural and muscle-rich tissues. Biochem Biophys Res Commun 1994, 198:637-645.

50. Tachikawa M, Fujinawa J, Takahashi M, Kasai Y, Fukaya M, Sakai K, Yamazaki M, Tomi M, Watanabe M, Sakimura K, et al: Expression and possible role of creatine transporter in the brain and at the bloodcerebrospinal fluid barrier as a transporting protein of guanidinoacetate, an endogenous convulsant. J Neurochem 2008, 107:768-778.

51. Tachikawa M, Kasai Y, Takahashi M, Fujinawa J, Kitaichi K, Terasaki T, Hosoya K: The blood-cerebrospinal fluid barrier is a major pathway of cerebral creatinine clearance: involvement of transporter-mediated process. J Neurochem 2008, 107:432-442.

52. Shojaiefard M, Christie DL, Lang F: Stimulation of the creatine transporter SLC6A8 by the protein kinases SGK1 and SGK3. Biochem Biophys Res Commun 2005, 334:742-746.
53. Dodd JR, Christie DL: Selective amino acid substitutions convert the creatine transporter to a gamma-aminobutyric acid transporter. J Biol Chem 2007, 282:15528-15533.

54. Dominy J Jr, Thinschmidt JS, Peris J, Dawson R Jr, Papke RL: Taurineinduced long-lasting potentiation in the rat hippocampus shows a partial dissociation from total hippocampal taurine content and independence from activation of known taurine transporters. Neurochem 2004, 89:1195-1205.

55. Liu QR, Lopez-Corcuera B, Mandiyan S, Nelson H, Nelson N: Molecular characterization of four pharmacologically distinct gamma-aminobutyric acid transporters in mouse brain [corrected]. J Biol Chem 1993, 268:2106-2112

56. Tachikawa M, Kasai Y, Yokoyama R, Fujinawa J, Ganapathy V, Terasaki T, Hosoya K: The blood-brain barrier transport and cerebral distribution of guanidinoacetate in rats: involvement of creatine and taurine transporters. J Neurochem 2009, 111:499-509.

57. Tomi M, Tajima A, Tachikawa M, Hosoya K: Function of taurine transporter (Slc6a6/TauT) as a GABA transporting protein and its relevance to GABA transport in rat retinal capillary endothelial cells. Biochim Biophys Acta 2008, 1778:2138-2142.

58. Urakami Y, Kimura N, Okuda M, Inui K: Creatinine transport by basolateral organic cation transporter hOCT2 in the human kidney. Pharm Res 2004, 21:976-981.

59. Kimura N, Masuda S, Katsura T, Inui K: Transport of guanidine compounds by human organic cation transporters, hOCT1 and hOCT2. Biochem Pharmacol 2009, 77:1429-1436.

60. Lightman SL, Palestine AG, Rapoport SI, Rechthand E: Quantitative assessment of the permeability of the rat blood-retinal barrier to small water-soluble non-electrolytes. J Physiol 1987, 389:483-490.

61. Sora I, Richman J, Santoro G, Wei H, Wang Y, Vanderah T, Horvath R, Nguyen M, Waite $S$, Roeske WR, et al: The cloning and expression of a human creatine transporter. Biochem Biophys Res Commun 1994, 204:419-427.

62. Guimbal C, Kilimann MW: A Na(+)-dependent creatine transporter in rabbit brain, muscle, heart, and kidney. cDNA cloning and functional expression. J Biol Chem 1993, 268:8418-8421.

63. Belanger M, Asashima T, Ohtsuki S, Yamaguchi $H$, Ito S, Terasaki T: Hyperammonemia induces transport of taurine and creatine and suppresses claudin-12 gene expression in brain capillary endothelial cells in vitro. Neurochem Int 2007, 50:95-101.

64. Tachikawa M, Fukaya M, Terasaki T, Ohtsuki S, Watanabe M: Distinct cellular expressions of creatine synthetic enzyme GAMT and creatine kinases uCK-Mi and CK-B suggest a novel neuron-glial relationship for brain energy homeostasis. Eur J Neurosci 2004, 20:144-160.

65. Braissant $\mathrm{O}$, Henry H, Loup M, Eilers B, Bachmann C: Endogenous synthesis and transport of creatine in the rat brain: an in situ hybridization study. Brain Res Mol Brain Res 2001, 86:193-201.

66. Balestrino M, Rebaudo R, Lunardi G: Exogenous creatine delays anoxic depolarization and protects from hypoxic damage: dose-effect relationship. Brain Res 1999, 816:124-130.

67. Brewer GJ, Wallimann TW: Protective effect of the energy precursor creatine against toxicity of glutamate and beta-amyloid in rat hippocampal neurons. J Neurochem 2000, 74:1968-1978.

68. Klivenyi P, Ferrante RJ, Matthews RT, Bogdanov MB, Klein AM, Andreassen OA, Mueller G, Wermer M, Kaddurah-Daouk R, Beal MF: Neuroprotective effects of creatine in a transgenic animal model of amyotrophic lateral sclerosis. Nat Med 1999, 5:347-350.

69. Matthews RT, Ferrante RJ, Klivenyi P, Yang L, Klein AM, Mueller G, KaddurahDaouk R, Beal MF: Creatine and cyclocreatine attenuate MPTP neurotoxicity. Exp Neurol 1999, 157:142-149.

70. Matthews RT, Yang L, Jenkins BG, Ferrante RJ, Rosen BR, Kaddurah-Daouk R, Beal MF: Neuroprotective effects of creatine and cyclocreatine in animal models of Huntington's disease. J Neurosci 1998, 18:156-163.

71. Schmidt A, Marescau B, Boehm EA, Renema WK, Peco R, Das A, Steinfeld R, Chan S, Wallis J, Davidoff $M$, et al: Severely altered guanidino compound levels, disturbed body weight homeostasis and impaired fertility in a mouse model of guanidinoacetate $\mathrm{N}$-methyltransferase (GAMT) deficiency. Hum Mol Genet 2004, 13:905-921.

72. Torremans A, Marescau B, Possemiers I, Van Dam D, D'Hooge R, Isbrandt D, De Deyn PP: Biochemical and behavioural phenotyping of a mouse model for GAMT deficiency. J Neurol Sci 2005, 231:49-55. 
73. Neu A, Neuhoff H, Trube G, Fehr S, Ullrich K, Roeper J, Isbrandt D: Activation of $\mathrm{GABA}(\mathrm{A})$ receptors by guanidinoacetate: a novel pathophysiological mechanism. Neurobiol Dis 2002, 11:298-307.

74. Keep RF, Xiang J: Choroid plexus taurine transport. Brain Res 1996, 715:17-24.

75. Sijens $P E$, Verbruggen $K T$, Oudkerk $M$, van Spronsen FJ, Soorani-Lunsing RJ: $1 \mathrm{H}$ MR spectroscopy of the brain in $\mathrm{Cr}$ transporter defect. Mol Genet Metab 2005, 86:421-422.

76. Braissant O, Henry H: AGAT, GAMT and SLC6A8 distribution in the central nervous system, in relation to creatine deficiency syndromes: A review. J Inherit Metab Dis 2008, 31:230-239.

77. Verbruggen KT, Sijens PE, Schulze A, Lunsing RJ, Jakobs C, Salomons GS, van Spronsen FJ: Successful treatment of a guanidinoacetate methyltransferase deficient patient: findings with relevance to treatment strategy and pathophysiology. Mol Genet Metab 2007, 91:294-296.

78. Mancardi MM, Caruso U, Schiaffino MC, Baglietto MG, Rossi A, Battaglia FM, Salomons GS, Jakobs C, Zara F, Veneselli E, Gaggero R: Severe epilepsy in X-linked creatine transporter defect (CRTR-D). Epilepsia 2007, 48:1211-1213.

79. Rosenberg EH, Almeida LS, Kleefstra T, deGrauw RS, Yntema HG, Bahi N, Moraine C, Ropers HH, Fryns JP, deGrauw TJ, et al: High prevalence of SLC6A8 deficiency in X-linked mental retardation. Am J Hum Genet 2004, 75:97-105.

80. Rosenberg EH, Martinez Munoz C, Betsalel OT, van Dooren SJ, Fernandez M, Jakobs C, deGrauw TJ, Kleefstra T, Schwartz CE, Salomons GS: Functional characterization of missense variants in the creatine transporter gene (SLC6A8): improved diagnostic application. Hum Mutat 2007, 28:890-896.

81. Almeida LS, Salomons GS, Hogenboom F, Jakobs C, Schoffelmeer AN: Exocytotic release of creatine in rat brain. Synapse 2006, 60:118-123.

82. De Deyn PP, Vanholder R, Eloot S, Glorieux G: Guanidino compounds as uremic (neuro)toxins. Semin Dial 2009, 22:340-345.

83. De Deyn PP, Marescau B, Cuykens JJ, Van Gorp L, Lowenthal A, De Potter WP: Guanidino compounds in serum and cerebrospinal fluid of non-dialyzed patients with renal insufficiency. Clin Chim Acta 1987, 167:81-88

84. De Deyn PP, Marescau B, D'Hooge R, Possemiers I, Nagler J, Mahler C: Guanidino compound levels in brain regions of non-dialyzed uremic patients. Neurochem Int 1995, 27:227-237.

85. De Deyn PP, D'Hooge R, Van Bogaert PP, Marescau B: Endogenous guanidino compounds as uremic neurotoxins. Kidney Int Supp/ 2001, 78 S77-83.

86. $\mathrm{Ku} \mathrm{CP}$, Passow $\mathrm{H}$ : Creatine and creatinine transport in old and young human red blood cells. Biochim Biophys Acta 1980, 600:212-227.

87. Nakayama H, Kitaichi K, Ito Y, Hashimoto K, Takagi K, Yokoi T, Ozaki N, Yamamoto T, Hasegawa T: The role of organic cation transporter-3 in methamphetamine disposition and its behavioral response in rats. Brain Res 2007, 1184:260-269.

doi:10.1186/2045-8118-8-13

Cite this article as: Tachikawa and Hosoya: Transport characteristics of guanidino compounds at the blood-brain barrier and bloodcerebrospinal fluid barrier: relevance to neural disorders. Fluids and Barriers of the CNS 2011 8:13.

\section{Submit your next manuscript to BioMed Central and take full advantage of:}

- Convenient online submission

- Thorough peer review

- No space constraints or color figure charges

- Immediate publication on acceptance

- Inclusion in PubMed, CAS, Scopus and Google Scholar

- Research which is freely available for redistribution

Submit your manuscript at www.biomedcentral.com/submit
Ciomed Central 